\title{
Stem Cell Markers Predict the Response to Sorafenib in Patients with Hepatocellular Carcinoma
}

\author{
Bo Hyun Kim ${ }^{1,2}$, Joong-Won Park ${ }^{1,2}$, Jin Sook Kim², Sook-Kyung Lee ${ }^{2}$, and Eun Kyung Hong ${ }^{1}$ \\ ${ }^{1}$ Center for Liver Cancer, and ${ }^{2}$ Common Cancer Branch, Division of Clinical Research, Research Institute, National Cancer Center, Goyang; \\ Korea
}

Background/Aims: Sorafenib remains the only approved molecular targeted agent for hepatocellular carcinoma (HCC); however, reliable biomarkers that predict its efficacy are still lacking. The aim of this study was to explore whether cancer stem cell (CSC) markers have a predictive role with regard to the sorafenib response in HCC patients. Methods: We enrolled 47 patients with HCC for whom tumor samples obtained before starting sorafenib treatment were available. RNA was extracted from formalin-fixed, paraffin-embedded samples, and real-time polymerase chain reaction was used to quantify mRNA expression of the CSC genes EpCAM, CD13, CK8, CD24, CD44, CD90, CD133, SALL4, ALDH1A1, $A L B$, and AFP. Results: Of 47 patients, $14.9 \%$ and $74.5 \%$ had vascular invasion and extrahepatic spread, respectively. Patients with low CD133 expression tended to have longer progression-free survival (PFS) than those with high CD133 expression (5.5 months vs 4.0 months), although without statistical significance. The expression levels of other markers were not associated with PFS. When examining markers in combination, patients with high $C D 133$ and $C D 90$ expression had shorter PFS rates than those with low expression (2.7 months vs 5.5 months; $p=0.04$ ). Patients with low CD133 and EpCAM expression demonstrated better PFS than those with high expression (7.0 months vs 4.2 months; $p=0.04)$. Multivariable analysis indicated that an Eastern Cooperative Oncology Group performance status score of 1 and high CD133/CD90 expression were significantly associated with shorter PFS. Conclusions: Overexpression of the CSC markers CD133 and CD90 in HCC was associated with poorer response to sorafenib. These two genes may serve as predictive biomarkers for sorafenib therapy. (Gut Liver 2019;13:342-348)
Key Words: Biomarkers; Cancer stem cells; Carcinoma, hepatocellular; Prognosis; Sorafenib

\section{INTRODUCTION}

Sorafenib is the first-line treatment option for patients with advanced hepatocellular carcinoma (HCC) who are unlikely to benefit from resection, transplantation, and other locoregional treatments; indeed, two large phase III clinical trials demonstrated a survival advantage with this drug. ${ }^{1,2}$ However, responses to sorafenib therapy vary among patients. Given the adverse effects and relatively high costs of sorafenib therapy, the identification of patients more likely to benefit from sorafenib therapy is necessary.

Numerous studies have revealed predictive factors for sorafenib therapy outcomes. The SHARP study, which aimed to assess potentially useful predictive biomarkers, showed that high baseline soluble c-KIT and low hepatocyte growth factor levels were independent predictors of survival in patients receiving sorafenib; however, the predictive trends were not significant. ${ }^{3}$ Early alpha-fetoprotein (AFP) expression, a des- $\gamma$ carboxyprothrombin response, and the development of hypertension or diarrhea may also predict the therapeutic efficacy of sorafenib therapy. ${ }^{4-9}$ Although additional studies exist, the results are inconsistent.

Accumulating evidence suggests that a subset of cancer cells with stem cell properties, referred to as cancer stem cells (CSCs), is capable of self-renewal and differentiation. ${ }^{10}$ CSCs are reported to be resistant to chemotherapy and to contribute to tumor persistence and relapse. ${ }^{11,12}$ Liver CSCs can be identified through certain cell surface markers, including CD133, EpCAM, CD90, CD44, 0V6, CD13, CD24, DLK1, $\alpha 2 \delta 1$, ICAM-1 and CD47. ${ }^{13}$ Recent studies have also found that the expression of CSC markers

Correspondence to: Joong-Won Park (https://orcid.org/0000-0001-9972-0494)

Center for Liver Cancer, National Cancer Center, 323 Ilsan-ro, llsandong-gu, Goyang 10408, Korea

Tel: +82-31-920-1605, Fax: +82-31-920-1520, E-mail: jwpark@ncc.re.kr

Received on August 7, 2018. Revised on September 18, 2018. Accepted on September 24, 2018. Published online April 17, 2019 pISSN 1976-2283 eISSN 2005-1212 https://doi.org/10.5009/gnl18345

(a) This is an Open Access article distributed under the terms of the Creative Commons Attribution Non-Commercial License (http://creativecommons.org/licenses/by-nc/4.0) which permits unrestricted non-commercial use, distribution, and reproduction in any medium, provided the original work is properly cited. 
is associated with HCC prognosis. An in vitro study demonstrated that the downregulation of CD44 protected HCC cells from sorafenib-induced apoptosis. ${ }^{14}$ In addition, high CD133 expression in HCC tissues was reported to be correlated with a poor response to sorafenib. ${ }^{15}$ Therefore, we performed this study to explore the role of CSC markers in predicting the sorafenib response in HCC patients.

\section{MATERIALS AND METHODS}

\section{Patients}

A total of 620 consecutive HCC patients were treated with sorafenib at the Center for Liver Cancer, National Cancer Center (Goyang, Korea) between June 2007 and March 2012. Of these patients, 417 were administered sorafenib for 6 weeks or longer. Patients were enrolled in this study if they met the following criteria: (1) they were treated with sorafenib for 6 weeks or longer; (2) pathological specimens extracted before commencement of sorafenib therapy were available; and (3) they discontinued sorafenib because of tumor progression or adverse events. Those who discontinued sorafenib because of economic burdens or other nonmedical problems were excluded. In addition, those who did not provide consent for the use of their specimens for genetic research were excluded. Patients were also excluded due to technical failure of RNA extraction and quantitative real-time polymerase chain reaction (RT-PCR) on their samples. Fortyseven patients were ultimately enrolled in this study (Fig. 1).

The diagnosis of HCC was based on histology and/or clinicoradiological evidence according to the Korean practice guidelines for HCC. ${ }^{16-18}$ The noninvasive diagnostic criteria established by these guidelines include the presence of one or more risk factors (i.e., hepatitis B virus, hepatitis C virus, or cirrhosis), typical enhancement on the arterial phase as well as washout in the delayed portal/venous phase in dynamic liver imaging (via methods such as dynamic spiral computed tomography) or contrast-enhanced dynamic magnetic resonance imaging, and/ or an elevated serum AFP level. Sorafenib was administered to patients with vascular invasion or extrahepatic spread and to those whose disease was not amenable to locoregional therapy.

Relevant data on clinical and tumor characteristics were extracted retrospectively from medical records. This study was conducted in accordance with the Declaration of Helsinki and International Conference on Harmonization-Good Clinical Practice. Moreover, this study was approved by the Institutional Review Board at the National Cancer Center (IRB number: NCC2016-0247); the requirement for written informed consent was waived.

\section{Outcomes and follow-up}

Patients were followed from the date of sorafenib initiation to the date of death or the last follow-up visit. Progression-free survival (PFS) was calculated from the date of sorafenib initiation to the date of first-documented disease progression or the date of death. Overall survival (OS) was calculated from the date of sorafenib initiation to the date of death or the date last seen alive. The response to treatment was assessed every 6 to 8 weeks according to the modified Response Evaluation Criteria in Solid Tumors for HCC. ${ }^{19}$

\section{RNA extraction from formalin-fixed paraffin-embedded HCC tissues}

Five 4- $\mu \mathrm{m}$ sections were cut from formalin-fixed paraffinembedded (FFPE) HCC tissues and deparaffinized. Total RNA was extracted using an RNeasy FFPE Kit (Qiagen, Valencia, CA, USA) according to the manufacturer's instructions. The RNA concentration was determined using a NanoDrop 2000 spectrophotometer (Thermo Fisher Scientific, Waltham, MA, USA).

\section{Quantitative RT-PCR}

One microgram of extracted RNA was reverse transcribed using a RevertAid First Strand cDNA Synthesis Kit (Thermo Fisher Scientific). Quantitative RT-PCR was performed using Taq-

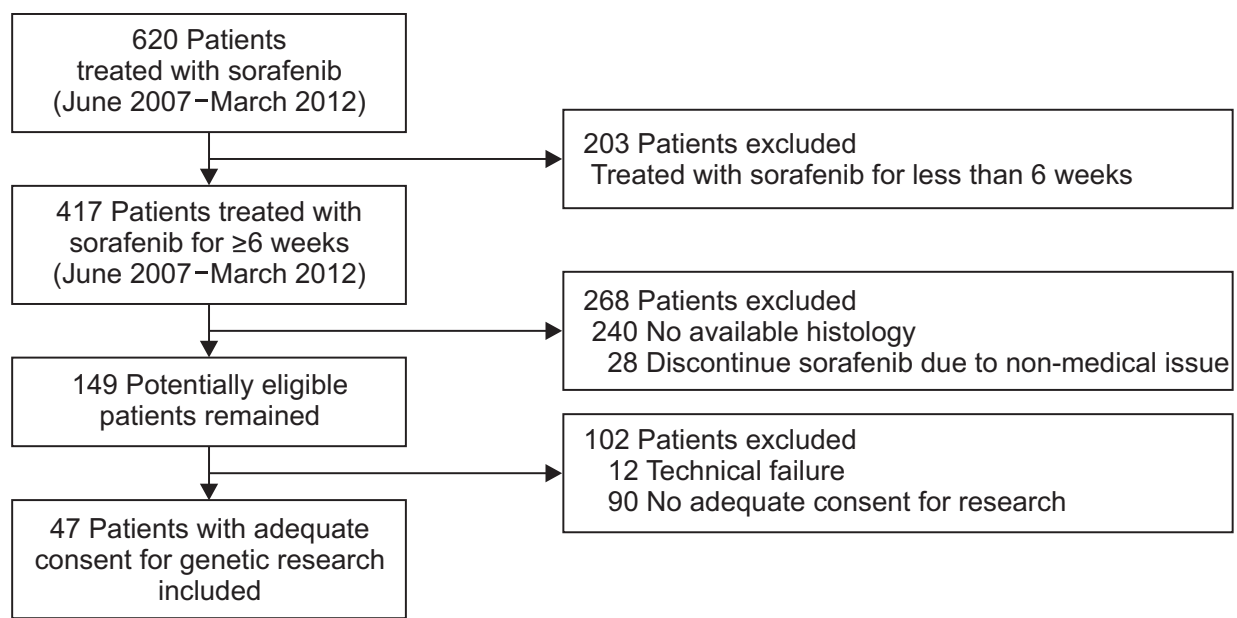

Fig. 1. Patient flow diagram. 
$\mathrm{Man}^{\circledR}$ gene expression master mix and the following TaqMan ${ }^{\circledR}$ gene expression probes: EpCAM (Hs00158980_m1), CK8/KRT8 (Hs01670053_m1), ALB (Hs00910225_m1), AFP (Hs01040598_ m1), GAPDH (Hs00266705_g1), CD13/ANPEP (Hs00174265_ m1), CD24 (Hs03044178_g1), CD44 (Hs01075856_m1), CD90/ THY1 (Hs00174816_m1), CD133/PROM1 (Hs01009250_m1), SALL4 (Hs00360675_m1), and ALDH1A1 (Hs00167445_m1) (Applied Biosystems, Foster City, CA, USA). The delta delta $\mathrm{CT}(\Delta \Delta \mathrm{CT})$ method was used to analyze the RT-PCR results. A QuantStudio $^{\text {TM }} 7$ Flex Real-Time PCR Systems instrument (Applied Biosystems) was used for RT-PCR. Relative gene expression was calculated using the $2^{-\Delta \Delta \mathrm{CT}}$ method.

\section{Statistical analysis}

All patients who met the eligibility criteria at baseline were included in the analyses. Continuous variables are expressed as medians and interquartile ranges. The total number of patients for each parameter varied because some data points were not available for every patient. Survival probabilities were estimated by the Kaplan-Meier method and compared by the log-rank test. Differences in survival were tested by using the log-rank test. Univariate and multivariable analyses were performed using the Cox proportional hazards model to identify significant variables related to PFS. Variables that were significant in the univariate analysis $(p \leq 0.05)$ were subjected to multivariable analysis using the backward elimination method. All statistical analyses in this study were performed using STATA software version 12.0 (StataCorp LP, College Station, TX, USA). All reported p-values are two-sided; p-values $<0.05$ were considered statistically significant.

\section{RESULTS}

\section{Patient characteristics}

Forty-seven patients were eligible for this study (Fig. 1). The median age was 55, and the predominant cause of liver disease was hepatitis B virus (63.6\%), as shown in Table 1. Three patients had combined HCC and cholangiocarcinoma. Vascular invasion was present in seven patients (14.9\%); extrahepatic spread, in 35 (74.5\%). Sixteen patients (34.0\%) had extrahepatic spread without viable intrahepatic lesions. The median duration of sorafenib administration was 123 days (interquartile range, 75.5 to 168.5$)$. Forty patients (85.1\%) showed progressive disease with sorafenib therapy. The median PFS was 4.9 months (95\% confidence interval [CI], 3.7 to 6.2 months) and the median OS was 9.7 months (95\% CI, 6.4 to 13.0 months).

\section{Association of CSC marker expression with response to sorafenib}

All specimens were obtained by surgical resection. When evaluated according to the Edmonson-Steiner grading system, 27 and nine specimens showed grade III and IV carcinoma, respectively. Expression of the CSC markers EpCAM, CD13, CD24, CD44, CD90, CD133, CK8, SALL4, ALDH1A1, ALB, and $A F P$ was assessed in 47 patients using quantitative RT-PCR (Supplementary Fig. 1). For each marker, the median expression level was chosen as the cutoff value for separating the highexpression group from the low-expression group.

Patients with high CD133 expression (CD133-high) showed shorter PFS than those with low expression (CD133-low); however, the difference was not statistically significant (4.0 months vs 5.5 months, respectively; hazard ratio [HR], 1.97) (Table 2, Fig. 2).

To further investigate the utility of CSC markers as predictive factors, we examined combinations of CD133 plus other markers with respect to PFS. Patients with a CD133-high/CD90high status demonstrated shorter PFS than those without high expression of both $C D 133$ and $C D 90$ (2.7 months vs 5.5 months, respectively; HR, 2.35; p=0.04) (Supplementary Table 1, Fig. 3A). However, patients with a CD133-low/EpCAM-low status had longer PFS than those without low expression of both $C D 133$ and EpCAM (7.0 months vs 4.2 months, respectively; HR, 0.31;

Table 1. Baseline Characteristics of the Study Population ( $n=47)$

\begin{tabular}{|c|c|}
\hline Characteristic & Value \\
\hline \multicolumn{2}{|l|}{ Clinical } \\
\hline Age, yr & $55(49-63)$ \\
\hline Male sex & $38(80.9)$ \\
\hline \multicolumn{2}{|l|}{ Etiology } \\
\hline HBV/HCV/alcoholic/others & $34(72.3) / 2(4.3) / 4(8.5) / 7(14.9$ \\
\hline \multicolumn{2}{|l|}{ Performance status } \\
\hline ECOG 0/ECOG 1 & $29(61.7) / 18(38.3)$ \\
\hline Cirrhosis & $28(59.6)$ \\
\hline \multicolumn{2}{|l|}{ Child-Turcotte-Pugh class } \\
\hline $\mathrm{A} / \mathrm{B}$ & $42(89.4) / 5(10.6)$ \\
\hline MELD score & $9.1(7.8-10.4)$ \\
\hline $\begin{array}{l}\text { Duration of sorafenib treatment, } \\
\text { day }\end{array}$ & $123(75.5-168.5)$ \\
\hline \multicolumn{2}{|l|}{ Tumor characteristics } \\
\hline \multicolumn{2}{|l|}{ Pathology } \\
\hline $\mathrm{HCC}$ & 44 (93.6) \\
\hline Combined HCC/CCC & $3(6.4)$ \\
\hline \multicolumn{2}{|l|}{ BCLC stage } \\
\hline $\mathrm{A} / \mathrm{B} / \mathrm{C}$ & $2(4.3) / 9(19.1) / 36(76.6)$ \\
\hline Vascular invasion & 7 (14.9) \\
\hline Extrahepatic spread & $35(74.5)$ \\
\hline Extrahepatic spread only & $16(34.0)$ \\
\hline $\mathrm{AFP}, \mathrm{ng} / \mathrm{mL}$ & $23.6(4.7-186.7)$ \\
\hline
\end{tabular}

Data are presented as the median (interquartile range) or number (\%). $\mathrm{HBV}$, hepatitis B virus; HCV, hepatitis C virus; ECOG, Eastern Cooperative Oncology Group; MELD, Model for End-Stage Liver Disease; HCC, hepatocellular carcinoma; CCC, cholangiocarcinoma; BCLC, Barcelona Clinic Liver Cancer; AFP, alpha-fetoprotein. 
Table 2. Progression-Free Survival According to Cancer Stem Cell Marker Expression

\begin{tabular}{|c|c|c|c|c|c|}
\hline \multicolumn{2}{|c|}{ CSC marker } & \multirow{2}{*}{$\begin{array}{c}\text { Progression-free survival (mo) } \\
4.2(1.3-7.2)\end{array}$} & \multirow{2}{*}{$\begin{array}{l}\mathrm{HR} \\
1\end{array}$} & \multirow[t]{2}{*}{ 95\% CI } & \multirow[t]{2}{*}{$p$-value } \\
\hline ЕрСАM & Low & & & & \\
\hline & High & $4.7(3.6-5.7)$ & 1.10 & $0.58-2.10$ & 0.77 \\
\hline \multirow[t]{2}{*}{ CD13 } & Low & $4.2(2.5-6.0)$ & 1 & & \\
\hline & High & $4.9(4.1-5.8)$ & 1.09 & $0.60-1.98$ & 0.77 \\
\hline \multirow[t]{2}{*}{ CD24 } & Low & $4.2(3.1-5.3)$ & 1 & & \\
\hline & High & $4.5(1.1-7.9)$ & 0.96 & $0.45-2.05$ & 0.91 \\
\hline \multirow[t]{2}{*}{$C D 44$} & Low & $4.0(2.6-5.4)$ & 1 & & \\
\hline & High & $4.7(2.9-6.4)$ & 1.15 & $0.57-2.34$ & 0.69 \\
\hline \multirow[t]{2}{*}{ CD90 } & Low & $5.4(3.4-7.4)$ & 1 & & \\
\hline & High & $4.5(2.4-6.5)$ & 1.07 & $0.59-1.94$ & 0.82 \\
\hline \multirow[t]{2}{*}{ CD133 } & Low & $5.5(3.9-7.0)$ & 1 & & \\
\hline & High & $4.0(1.7-6.3)$ & 1.97 & $0.91-4.30$ & 0.09 \\
\hline \multirow[t]{2}{*}{ CK8 } & Low & $2.6(0.5-4.7)$ & 1 & & \\
\hline & High & $4.5(2.4-6.5)$ & 0.70 & $0.24-2.08$ & 0.52 \\
\hline \multirow[t]{2}{*}{ SALL4 } & Low & $3.9(1.0-6.9)$ & 1 & & \\
\hline & High & $5.4(3.4-7.4)$ & 0.73 & $0.38-1.41$ & 0.35 \\
\hline \multirow[t]{2}{*}{$A L D H 1 A 1$} & Low & $4.9(3.5-6.4)$ & 1 & & \\
\hline & High & $4.7(3.2-6.1)$ & 0.96 & $0.53-1.74$ & 0.89 \\
\hline \multirow[t]{2}{*}{ Albumin } & Low & $5.4(3.2-7.6)$ & 1 & & \\
\hline & High & $4.7(3.4-5.9)$ & 1.15 & $0.62-2.11$ & 0.66 \\
\hline \multirow[t]{2}{*}{$A F P$} & Low & $4.9(3.0-7.3)$ & 1 & & \\
\hline & High & $4.1(3.0-5.2)$ & 1.32 & $0.69-2.54$ & 0.41 \\
\hline
\end{tabular}

Data are presented as the median (95\% CI).

CSC, cancer stem cell; HR, hazard ratio; CI, confidence interval; AFP, alpha-fetoprotein.

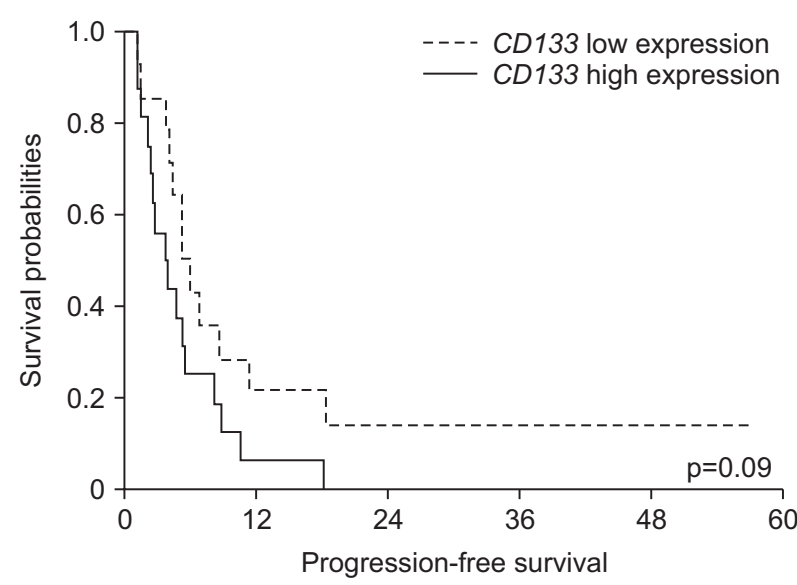

Fig. 2. Progression-free survival in hepatocellular carcinoma patients receiving sorafenib according to $C D 133$ expression.

p=0.04) (Supplementary Table 1, Fig. 3B).

Univariate analysis revealed that among the clinical features tested, Eastern Cooperative Oncology Group (ECOG) performance status and vascular invasion were associated with PFS (Table 3). Among the variables selected for multivariable analysis (ECOG performance status, vascular invasion, CD133high/CD90-high status, and CD133-low/EpCAM-low status), an
Table 3. Univariate and Multivariable Analysis for Progression-Free Survival

\begin{tabular}{llc}
\hline \multicolumn{1}{c}{ Variable } & HR $(95 \%$ CI $)$ & p-value \\
\hline Univariate analysis & & \\
ECOG 1 & $1.86(0.997-3.45)$ & 0.05 \\
Child-Turcotte-Pugh class B & $1.62(0.63-4.20)$ & 0.32 \\
Vascular invasion & $2.93(1.26-6.83)$ & 0.01 \\
Extrahepatic spread & $1.07(0.54-2.09)$ & 0.85 \\
Extrahepatic spread only & $0.65(0.34-1.24)$ & 0.19 \\
Multivariable analysis & & \\
ECOG 1 & $3.08(1.27-7.47)$ & 0.01 \\
CD133-high/CD90-high & $2.97(1.24-7.07)$ & 0.01 \\
\hline
\end{tabular}

HR, hazard ratio; CI, confidence interval; ECOG, Eastern Cooperative Oncology Group.

ECOG performance status of 1 and a CD133-high/CD90-high status were significantly associated with shorter PFS (Table 3).

\section{Survival outcome according to CSC marker expression}

The median OS of patients with high expression of both CD133 and CD90 was 9.4 months (95\% CI, 1.1 to 17.8 months), shorter than the 14.0 months (95\% CI, 7.1 to 20.8 months) in 

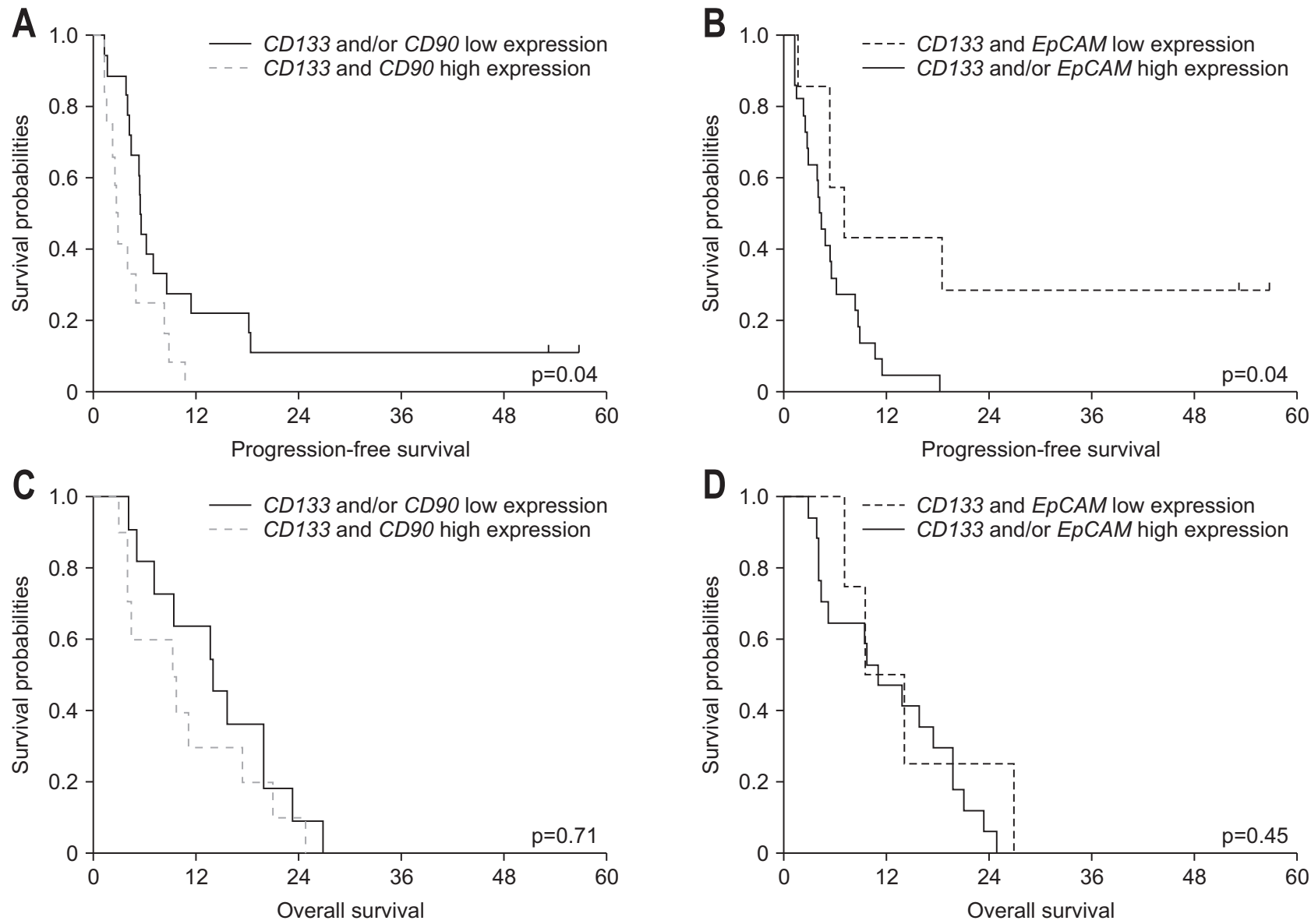

Fig. 3. Survival outcome in hepatocellular carcinoma patients receiving sorafenib according to CD133, CD90, and EpCAM expression. (A) Progression-free survival according to CD133 and CD90 expression. (B) Progression-free survival according to CD133 and EpCAM expression. (C) 0verall survival according to CD133 and CD90 expression. (D) Overall survival according to CD133 and EpCAM expression.

of those who did not have high expression for both $C D 133$ and CD90 ( $\mathrm{p}=0.71$; Fig. 3C). The median OS of patients with low expression of both CD133 and EpCAM was 11.1 months (95\% CI, 5.2 to 16.9 months), longer than the 9.4 months (95\% CI, 2.6 to 16.2 months) in those who did not have low expression for both CD133 and EpCAM (p=0.45; Fig.3D). However, these differences were not statistically significant.

\section{DISCUSSION}

Many studies have suggested that CSCs are responsible for resistance to various chemotherapeutics in cancers $;^{20}$ however, the underlying mechanisms by which CSCs elicit chemoresistance remain largely unknown. One hypothesis for the chemoresistance mechanism is that CSCs are located in niches where they are shielded from drugs or can regulate the tumor microenvironment and exposure through drug efflux. Other theories are that CSCs can inactivate drugs in the cytoplasm or that they possess a robust DNA damage response and DNA repair mechanisms that involve the upregulation of prosurvival or antiapoptotic signaling pathways. ${ }^{20}$ Active drug efflux is mediated by several ATPbinding cassette transporters such as multidrug resistance pro- tein 1 (MDR1/ABCB1), multidrug resistance-associated protein 1 (MRP1/ABCC1), and breast cancer resistance protein (BCRP/ ABCG2). ${ }^{20,21}$

In the present study, we showed that CD133 expression was related to the response to sorafenib; however, the association was not statistically significant for CD133 alone. However, a CD133-high/CD90-high status was negatively associated but a CD133-low/EpCAM-low status was positively associated with the sorafenib response. Among the clinical factors and CSC markers investigated, an ECOG performance status of 1 and a CD133high/CD90-high status were significantly associated with a poor response to sorafenib. CD133-high/CD90-high and CD133-low/ EpCAM-low statuses also tended to be associated with OS; however, neither association was statistically significant.

CD133 was originally identified as a member of the prominin family of pentaspan transmembrane glycoproteins ( 5 transmembrane domains) and is also known as prominin- $1 .^{22}$ CD133 is usually recognized as a surface antigen on hematopoietic progenitor cells; however, its biological function is largely unknown. CD133 is also regarded as a CSC marker in various cancers such as brain, colon, non-small cell lung, laryngeal, and liver cancers. $^{23-27}$ In HCC, high expression of CD133 has been correlated 
with increased tumor grade, advanced tumor stage, poor OS, and a high recurrence rate. ${ }^{28,29}$ Moreover, CD133 has been implicated in chemoresistance and radioresistance in glioblastoma, lung cancer, colon cancer, and HCC. ${ }^{30-34}$ Ma et al..$^{33}$ showed that CD133+ HCC cells were more resistant than CD133- HCC cells to chemotherapeutic agents such as doxorubicin and 5-fluorouracil; this resistance was mediated by the preferential activation of the Akt/PKB and Bcl-2-mediated cell survival pathways.

CD90 encodes a glycophosphatidylinositol-anchored cell surface protein that is involved in cell-cell and cell-matrix interactions and is also known as THY $1 .^{35}$ This protein is usually expressed on $\mathrm{T}$ cells, neurons, endothelial cells, mesenchymal stem cells, hematopoietic stem cells, and fibroblasts. ${ }^{36}$ CD90 is upregulated in cancers such as esophageal cancer, gastric cancer, and high-grade glioma ${ }^{37-39}$ and is associated with poor differentiation or high pathological grade in HCC. ${ }^{40,41}$ In addition, CD90 expression was associated with recurrence and predicted poor prognosis in HCC patients who underwent surgical resection. ${ }^{41,42}$ Moreover, CD90-positive esophageal cancer cells were chemoresistant to 5-fluorouracil and Taxotere. ${ }^{37}$ CD90-positive HCC cells are reportedly resistant to doxorubicin, and BCRP/ABCG2 expression can contribute to this chemoresistance. ${ }^{43,44}$

This study has some limitations. First, this study was a retrospective study in a cohort of 47 patients; the small sample size may have biased the results. Second, CSC markers were evaluated in pathological specimens, although blood-based biomarkers are usually preferred over tissue-based biomarkers because blood is more convenient and less invasive to obtain than tissue. In fact, pathological specimens were not available for some of the HCC patients since HCC can be diagnosed via noninvasive methods. Although liver biopsies have been considered confirmatory tests in small or atypical tumors, they have increasingly been considered a prerequisite for companion diagnostics in recent biomarker-driven clinical trials. ${ }^{45}$ Third, we were unable to explore the mechanisms linking high expression of CD133/ CD90 with the response to sorafenib. However, the expression of the CD133 or CD90 proteins may promote sorafenib resistance through the regulation of survival and apoptosis signaling pathways as well as through active drug efflux, as the results of previous studies suggest. $33,43,44$

Our results demonstrated that the level of CD133 and CD90 expression was associated with the clinical response to sorafenib. Such CSC markers may therefore be useful predictors of sorafenib therapy outcomes; further investigations of the implications of CSCs in HCC are warranted.

\section{CONFLICTS OF INTEREST}

No potential conflict of interest relevant to this article was reported.

\section{ACKNOWLEDGEMENTS}

This work was supported by a grant from the National Cancer Center, Korea (No. 1510520 and No. 1810031). The funders had no role in study design, data collection and analysis, decision to publish, or preparation of the manuscript. We thank Eun-Ah Cho, RN (Center for Liver Cancer, National Cancer Center) for greatly assisting with this study.

Author contributions: B.H.K., J.W.P. were responsible for the conception and design of the study, the acquisition, analysis, and interpretation of the data, and the drafting of the manuscript. J.S.K., S.K.L. were responsible for the acquisition of the data, and analysis of data. E.K.H. was responsible for the acquisition, analysis and interpretation of the data. All authors contributed to manuscript writing and final manuscript approval.

\section{REFERENCES}

1. Llovet JM, Ricci S, Mazzaferro V, et al. Sorafenib in advanced hepatocellular carcinoma. N Engl J Med 2008;359:378-390.

2. Cheng AL, Kang YK, Chen Z, et al. Efficacy and safety of sorafenib in patients in the Asia-Pacific region with advanced hepatocellular carcinoma: a phase III randomised, double-blind, placebocontrolled trial. Lancet Oncol 2009;10:25-34.

3. Llovet JM, Peña CE, Lathia CD, et al. Plasma biomarkers as predictors of outcome in patients with advanced hepatocellular carcinoma. Clin Cancer Res 2012;18:2290-2300.

4. Kuzuya T, Asahina Y, Tsuchiya K, et al. Early decrease in $\alpha$-fetoprotein, but not des- $\gamma$-carboxy prothrombin, predicts sorafenib efficacy in patients with advanced hepatocellular carcinoma. Oncology 2011;81:251-258.

5. Ueshima K, Kudo M, Takita M, et al. Des-gamma-carboxyprothrombin may be a promising biomarker to determine the therapeutic efficacy of sorafenib for hepatocellular carcinoma. Dig Dis 2011;29:321-325.

6. Kudo M, Ueshima K, Arizumi T. Real-life clinical practice with sorafenib in advanced hepatocellular carcinoma: a single-center experience. Dig Dis 2012;30:609-616.

7. Estfan B, Byrne M, Kim R. Sorafenib in advanced hepatocellular carcinoma: hypertension as a potential surrogate marker for efficacy. Am J Clin Oncol 2013;36:319-324.

8. Koschny R, Gotthardt D, Koehler C, Jaeger D, Stremmel W, Ganten TM. Diarrhea is a positive outcome predictor for sorafenib treatment of advanced hepatocellular carcinoma. Oncology 2013;84:613.

9. Bettinger D, Schultheiss M, Knüppel E, Thimme R, Blum HE, Spangenberg HC. Diarrhea predicts a positive response to sorafenib in patients with advanced hepatocellular carcinoma. Hepatology 2012;56:789-790.

10. Clevers $\mathrm{H}$. The cancer stem cell: premises, promises and challenges. Nat Med 2011;17:313-319.

11. O'Connor ML, Xiang D, Shigdar S, et al. Cancer stem cells: 
a contentious hypothesis now moving forward. Cancer Lett 2014;344:180-187.

12. Clarke MF, Dick JE, Dirks PB, et al. Cancer stem cells: perspectives on current status and future directions: AACR Workshop on cancer stem cells. Cancer Res 2006;66:9339-9344.

13. Cheng Z, Li X, Ding J. Characteristics of liver cancer stem cells and clinical correlations. Cancer Lett 2016;379:230-238.

14. Fernando J, Malfettone A, Cepeda EB, et al. A mesenchymallike phenotype and expression of CD44 predict lack of apoptotic response to sorafenib in liver tumor cells. Int $\mathrm{J}$ Cancer 2015;136:E161-E172.

15. Hagiwara S, Kudo M, Nagai T, et al. Activation of JNK and high expression level of $\mathrm{CD} 133$ predict a poor response to sorafenib in hepatocellular carcinoma. Br J Cancer 2012;106:1997-2003.

16. Korean Liver Cancer Study Group (KLCSG); National Cancer Center, Korea (NCC). 2014 KLCSG-NCC Korea practice guideline for the management of hepatocellular carcinoma. Gut Liver 2015;9:267-317.

17. Yu SJ. A concise review of updated guidelines regarding the management of hepatocellular carcinoma around the world: 20102016. Clin Mol Hepatol 2016;22:7-17.

18. Korean Liver Cancer Study Group and National Cancer Center, Korea. Practice guidelines for management of hepatocellular carcinoma 2009. Korean J Hepatol 2009;15:391-423.

19. Lencioni R, Llovet JM. Modified RECIST (mRECIST) assessment for hepatocellular carcinoma. Semin Liver Dis 2010;30:52-60.

20. Zhao J. Cancer stem cells and chemoresistance: the smartest survives the raid. Pharmacol Ther 2016;160:145-158.

21. Gottesman MM, Fojo T, Bates SE. Multidrug resistance in cancer: role of ATP-dependent transporters. Nat Rev Cancer 2002;2:48-58.

22. Ma S. Biology and clinical implications of CD133(+) liver cancer stem cells. Exp Cell Res 2013;319:126-132.

23. Singh SK, Hawkins C, Clarke ID, et al. Identification of human brain tumour initiating cells. Nature 2004;432:396-401.

24. Ricci-Vitiani L, Lombardi DG, Pilozzi E, et al. Identification and expansion of human colon-cancer-initiating cells. Nature 2007;445:111-115.

25. Tirino V, Camerlingo R, Franco R, et al. The role of CD133 in the identification and characterisation of tumour-initiating cells in non-small-cell lung cancer. Eur J Cardiothorac Surg 2009;36:446453.

26. Qiu HO, Wang H, Che N, et al. Identification and characterization of CD133(pos) subpopulation cells from a human laryngeal cancer cell line. Med Sci Monit 2016;22:1146-1151.

27. Ma S, Chan KW, Hu L, et al. Identification and characterization of tumorigenic liver cancer stem/progenitor cells. Gastroenterology 2007;132:2542-2556.

28. Song W, Li H, Tao K, et al. Expression and clinical significance of the stem cell marker CD133 in hepatocellular carcinoma. Int J Clin Pract 2008;62:1212-1218.

29. Ma S, Tang KH, Chan YP, et al. miR-130b Promotes CD133(+) liver tumor-initiating cell growth and self-renewal via tumor protein 53-induced nuclear protein 1. Cell Stem Cell 2010;7:694-707.

30. Liu G, Yuan X, Zeng Z, et al. Analysis of gene expression and chemoresistance of $\mathrm{CD} 133+$ cancer stem cells in glioblastoma. Mol Cancer 2006;5:67.

31. Liu YP, Yang CJ, Huang MS, et al. Cisplatin selects for multidrugresistant CD133+ cells in lung adenocarcinoma by activating Notch signaling. Cancer Res 2013;73:406-416.

32. Lee MR, Ji SY, Mia-Jan K, Cho MY. Chemoresistance of CD133(+) colon cancer may be related with increased survivin expression. Biochem Biophys Res Commun 2015;463:229-234.

33. Ma S, Lee TK, Zheng BJ, Chan KW, Guan XY. CD133+ HCC cancer stem cells confer chemoresistance by preferential expression of the Akt/PKB survival pathway. Oncogene 2008;27:1749-1758.

34. Piao LS, Hur W, Kim TK, et al. CD133+ liver cancer stem cells modulate radioresistance in human hepatocellular carcinoma. Cancer Lett 2012;315:129-137.

35. Rege TA, Hagood JS. Thy-1 as a regulator of cell-cell and cellmatrix interactions in axon regeneration, apoptosis, adhesion, migration, cancer, and fibrosis. FASEB J 2006;20:1045-1054.

36. Kumar A, Bhanja A, Bhattacharyya J, Jaganathan BG. Multiple roles of CD90 in cancer. Tumour Biol 2016;37:11611-11622.

37. Tang KH, Dai YD, Tong M, et al. A CD90(+) tumor-initiating cell population with an aggressive signature and metastatic capacity in esophageal cancer. Cancer Res 2013;73:2322-2332.

38. Zhu GC, Gao L, He J, et al. CD90 is upregulated in gastric cancer tissues and inhibits gastric cancer cell apoptosis by modulating the expression level of SPARC protein. Oncol Rep 2015;34:24972506.

39. He J, Liu Y, Zhu T, et al. CD90 is identified as a candidate marker for cancer stem cells in primary high-grade gliomas using tissue microarrays. Mol Cell Proteomics 2012;11:M111.010744.

40. Liu R, Shen Y, Nan K, et al. Association between expression of cancer stem cell markers and poor differentiation of hepatocellular carcinoma: a meta-analysis (PRISMA). Medicine (Baltimore) 2015;94:e1306.

41. Zhao RC, Zhou J, Chen KF, et al. The prognostic value of combination of CD90 and OCT4 for hepatocellular carcinoma after curative resection. Neoplasma 2016;63:288-298.

42. Guo Z, Li LQ, Jiang JH, Ou C, Zeng LX, Xiang BD. Cancer stem cell markers correlate with early recurrence and survival in hepatocellular carcinoma. World J Gastroenterol 2014;20:2098-2106.

43. Sukowati CH, Anfuso B, Torre G, Francalanci P, Crocè LS, Tiribelli C. The expression of CD90/Thy-1 in hepatocellular carcinoma: an in vivo and in vitro study. PLoS One 2013;8:e76830.

44. Jia Q, Zhang X, Deng T, Gao J. Positive correlation of Oct4 and ABCG2 to chemotherapeutic resistance in CD90(+)CD133(+) liver cancer stem cells. Cell Reprogram 2013;15:143-150.

45. Finn RS. The role of liver biopsy in hepatocellular carcinoma. Gastroenterol Hepatol (N Y) 2016;12:628-630. 